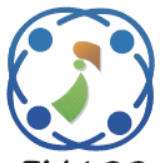

\title{
Acoustic Echo Cancellation Using PEVD Based Adaptive Kalman Filter
}

\author{
Mohanaprasad Kothandaraman $^{1 *}$ Jeet Kiran Pawani ${ }^{2} \quad$ Arulmozhivarman Pachaiyappan $^{2}$ \\ Sankar Ganesh Sankaran ${ }^{2}$
}

\author{
${ }^{1}$ School of Electronics Engineering, VIT University, Chennai, Tamil Nadu, 600127, India \\ ${ }^{2}$ School of Electronics Engineering, VIT University, Vellore, Tamil Nadu, 632014, India \\ * Corresponding author's Email: kmohanaprasad@vit.ac.in
}

\begin{abstract}
In this paper a PEVD (Polynomial Eigen Value Decomposition) based adaptive kalman filter is proposed for Acoustic Echo Cancellation (AEC) in the presence of noise. Acoustic echo is the phenomenon when the speaker listens to his own voice after some delay while speaking to his fellow mate on a call. The presence of acoustic coupling between the loudspeakers and the near-end microphone signal produces an undesired acoustic echo, which reduces the speech quality. Existing AEC system implemented adaptive kalman filter which results in less output efficiency in the noisy environment. In the proposed method, the near end speech is separated from the acoustic echo as well as from the surrounding noise by using PEVD based adaptive kalman filter. Initially mixed microphone signal is pre-processed by Polynomial Eigen Value Decomposition which strongly de-correlates the signal and also de-correlates out the noise. The pre-processed signal is passed through adaptive kalman filter for estimation of the acoustic echo. The efficiency of the output is calculated using Echo Return Loss Enhancement (ERLE). If the ERLE lies in the range of 30-40 dB indicates the good echo cancellation. The simulations show that the proposed PEVD based adaptive kalman method provides higher cancellation of echo. The simulations are being carried out in Matlab 2016 .
\end{abstract}

Keywords: Kalman filters, Acoustic echo cancellation, Polynomial eigen value decomposition, Echo return loss enhancement, Adaptive filter, Double talk detection.

\section{Introduction}

In the today's technological world, one of the most commonly used gadgets is a mobile phone or speaker phone or earphone to talk to one another. Though these devices provide us an excellent way of communication between people but the problem of acoustic echo always comes up which causes miserable amount of disturbance in our communication. In hands free terminals, the acoustic echo arises due to coupling between a far-end signal (loudspeaker) and microphone signal, which degrades the quality of speech.

The Acoustic Echo Cancellation (AEC) is employed by an adaptive filter which uses various adaptation algorithms [1] like LMS (Least Mean Square), NLMS (Normalized Least Mean Square), and RLS (Recursive Least Square) to estimate the echo signal. The estimated echo is subtracted from the microphone signal to cancel the echo [2-4]. The above adaptive filter fails in double talk situation where both the far-end and the near-end speech signals occur simultaneously. In this situation the near-end speech signal is included in the microphone signal along with the residual echo signal, which further degrades the speech quality. Once the double talk situation is recognized, the adaptive filter has to stop updating the filter coefficients, but it continues the comparison, allowing some part of echo to pass through. The double talk is identified by measuring the crosscorrelation coefficient vector between far-end speech and error signal using orthogonality theorem by $\mathrm{Ye}$ and $\mathrm{Wu}$ [5]. In this method it is difficult to predict the error signal. Gansler [6] proposed the coherence function that measured the similarity between far-end speech and near-end speech signal 
for double talk detection instead of error prediction. The coherence function needs to fix the threshold value to identify the double talk. A robust and reliable way to find double talk is to measure the correlation coefficient vector between the far-end speech and the microphone signal [7-8]. The efficiency of the echo cancellation is less in terms of ELRE. The improved efficiency with reduced computational complexity of double talk detection algorithm is achieved by normalizing the crosscorrelation vector between far-end speech and microphone signal [9]. In the time domain adaptive filter the convergence behavior is less. The convergence of the full band adaptive filter is further improved by using frequency domain adaptive algorithm combined with normalized crosscorrelation based double talk detector [10]. The decision rule of double talk detection has been made heuristic and simple by using generalized log likelihood ratio test [11-12]. The efficiency of the above techniques is increased by using frequency domain double talk detector based on the Gaussian Mixture Model (GMM) [13]. The above discussed double talk detection algorithms give only the partial solution to the echo cancellation problem due to noise added in a microphone signal.

R. E. Kalman [14] proposed a set of filtering technique, which has been used to the estimate unknown variables based on noisy observation using Bayesian approach. Enzner and Vary [15] proposed a frequency domain kalman filter for echo cancellation problem. The noise is not considered in the above method. The acoustic echo and noise cancellation of the generalized side lobe canceller was proposed by Tanan et al [16]. In this work they developed multichannel adaptive kalman filter (MCAKF) for estimating the echo and noise path in acoustic echo cancellation. Constantin paleologu et al [17] given soulution for stereophonic acoustic echo cancellation using widely linear general kalman filter. Recently, Ayush jain et al [18], implemented the Robust acoustic echo cancellation using kalman filter in double talk scenario and the efficiency is less than $30 \mathrm{~dB}$ in terms of ERLE (Echo Return Loss Enhancement). In all the above methods efficiency in terms of ERLE is not more than $30 \mathrm{~dB}$. According to ITU standards increase in ERLE increases the efficiency of the echo cancellation.

In this paper we propose a PEVD (Polynomial Eigen Value Decomposition) [19] based adaptive kalman filter to increase the efficiency of the AEC in terms of ELRE having more than $30 \mathrm{~dB}$ in the presence of noise. Firstly, pre-processing step called Polynomial Eigen Value Decomposition which will help us for strong de-correlation of the signal thoroughly and also remove some part of the noise from our signal. Then we will apply adaptive Kalman Filtering which will help us remove the acoustic echo and the residual noise which will be present in our communication.

The rest of the paper is organized as follows: Fundamentals of Acoustic Echo Cancellation is detailed in section 2, proposed method using PEVD adaptive kalman filter is discussed in section 3 , Implementation of proposed methodology is demonstrated in section 4 and conclusion is briefed in section 5 .

\section{Fundamentals of acoustics echo cancellation}

\subsection{Fundamentals of acoustic echo}

In acoustics and audio signal processing, an echo is a reflection of sound, arriving at the listener sometime after the original sound was heard. A single reflection of the sound source is called a true echo. The extra distance divided by the speed of sound is the time delay. Reverberation is the phenomenon in which, if so many multiple reflections arrive at a listener that they are unable to distinguish between them as there is a lot of delay. An echo can be explained as well as perfectly understood as a wave that has been reflected by an imperfection in the propagation travelling medium, and returns with sufficient delay and magnitude to be perceived. Echoes are reflected off hard surfaces or walls like mountains and privacy fences. Or even it can be said they are attenuated in the medium due to various different things in the medium.

When dealing with frequencies which are in the audible range, the human ear is not able to distinguish original sound's echo if the delay/attenuation is less than $1 / 15$ of a second. Thus, since the velocity/speed of sound is approximately $343 \mathrm{~m} / \mathrm{s}$ at a normal room temperature of about $25^{\circ} \mathrm{C}, 17.2 \mathrm{~m}$ is the maximum distance at which the reflecting object can be from the sound source at this temperature for an echo to be heard by a person at the source as human ears are not able to sense the echo.

\subsection{Formulation of adaptive filter for acoustic echo cancellation}

Echo cancellation and echo suppression are methods in telephony which help improve the speech and voice quality by preventing or reducing the echo from being created or removing it after it is 
already present or already formed. In addition to improving subjective quality as well as clarity of the speech and voice, this process definitely increases the capacity achieved through silence suppression or removal by preventing echo from traveling across a network.

Acoustic echo suppression (AES) and Acoustic echo cancellation (AEC) are commonly known methods, and more rarely called line echo cancellation (LEC). In some cases or rare cases, these terms are more precise or specific, as there are various different types and causes of formation of echo with unique characteristics, including acoustic echo which in broad terms is sounds from a loudspeaker recorded by a microphone after being reflected, which can vary substantially over time which causes huge problem in voice output and line echo which is explained as electrical impulses caused by, e.g., coupling between the, impedance mismatches, sending and receiving wires, reflections by electrical things, etc., which varies much less than acoustic echo. In practice, however, the same similar techniques are being used to treat/remove all types of echo, so an acoustic echo canceller is capable of cancelling both line echo as well as acoustic echo. "AEC" in particular and particularly is commonly used to refer to echo cancelers regardless of whether they were intended for line echo, acoustic echo, or both in general. Basic block diagram for Acoustic Echo Cancellation using Adaptive filter [20-22] is shown in Fig.1.

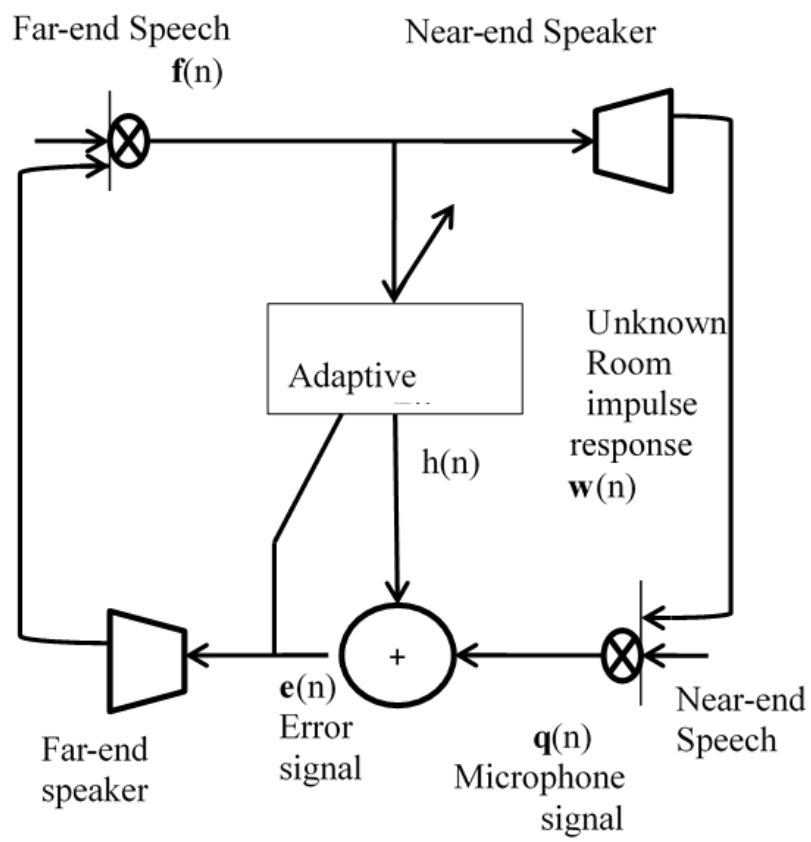

Figure.1 Basic block diagram of an Acoustic Echo Cancellation using Adaptive filter $\boldsymbol{f}(n)$ is the far-end speech signal, $\boldsymbol{v}(n)$ is the near-end speech signal, $\boldsymbol{q}(n)$ is the received microphone signal. It is given by

$$
\boldsymbol{q}(n)=\boldsymbol{h}(n)+\boldsymbol{v}(n)
$$

Where $\boldsymbol{h}(n)$ is acoustic echo and it is mathematically given as

$$
\boldsymbol{h}(n)=\sum_{m=0}^{M-1} w^{T} \boldsymbol{f}(n-m)
$$

$\boldsymbol{w}^{T}$ is the room impulse response in the receiver room. $\boldsymbol{h}^{\wedge}(n)$ is the estimated echo using adaptive filter, and $\boldsymbol{e}(n)$ is an error signal given by

$$
\boldsymbol{e}(n)=\boldsymbol{q}(n)+\widehat{\boldsymbol{h}}(n)
$$

\section{The proposed method using PEVD based adaptive kalman filter}

\subsection{Polynomial eigen value decomposition [23-24]}

The decomposition of the Eigen Values of a para-Hermitian polynomial matrix, $\boldsymbol{R}(z)$ belongs to $\mathrm{C}_{\mathrm{p} \times \mathrm{p}}$, is defined here as

$$
\boldsymbol{R}(z)=\widetilde{\boldsymbol{H}}(z) \boldsymbol{D}(z) \boldsymbol{H}(z)
$$

Where the polynomial matrix $\boldsymbol{H}(z)$ belongs to $\mathrm{C}_{\mathrm{p} \times \mathrm{p}}$ is paraunitary and the resulting polynomial matrix $\boldsymbol{D}(z)$ belongs to $\mathrm{C}_{\mathrm{pxp}}$ is diagonal. Note that the matrix to be diagonalise $\boldsymbol{R}(z)$ must be para-Hermitian, which means all coefficients of the matrix must satisfy $r_{j k}(t)$ $=r_{k j}(-t)$ for $j, k=1, \ldots . . p$ and for all values of the lag parameter $t$ belongs to $z$.

Given the para-Hermitian polynomial matrix $\boldsymbol{R}(z)$ belongs to $\mathrm{C}_{\mathrm{p} \times \mathrm{p}}$, the objective of the SBR2/PEVD algorithm [23] is to compute the paraunitary polynomial matrix $\boldsymbol{H}(z)$ such that

$$
\boldsymbol{H}(z) \boldsymbol{R}(z) \widetilde{\boldsymbol{H}}(z)=\boldsymbol{D}(z)
$$

Where the polynomial matrix $\boldsymbol{D}(z)$ belongs to $\mathrm{C}_{\mathrm{p} \times \mathrm{p}}$ is diagonal. The algorithm operates as an iterative process, where at each iteration an elementary paraunitary transformation is applied to both sides of the polynomial matrix $\boldsymbol{R}(z)$ designed to drive the two off-diagonal coefficients with the largest magnitude to zero. The paraunitary polynomial transformation matrix $\boldsymbol{H}(z)$ is therefore formulated as a series of elementary paraunitary matrices, i.e.

$$
\boldsymbol{H}(z)=\boldsymbol{G}_{i}(z) \ldots \ldots \cdot \boldsymbol{G}_{1}(z)
$$




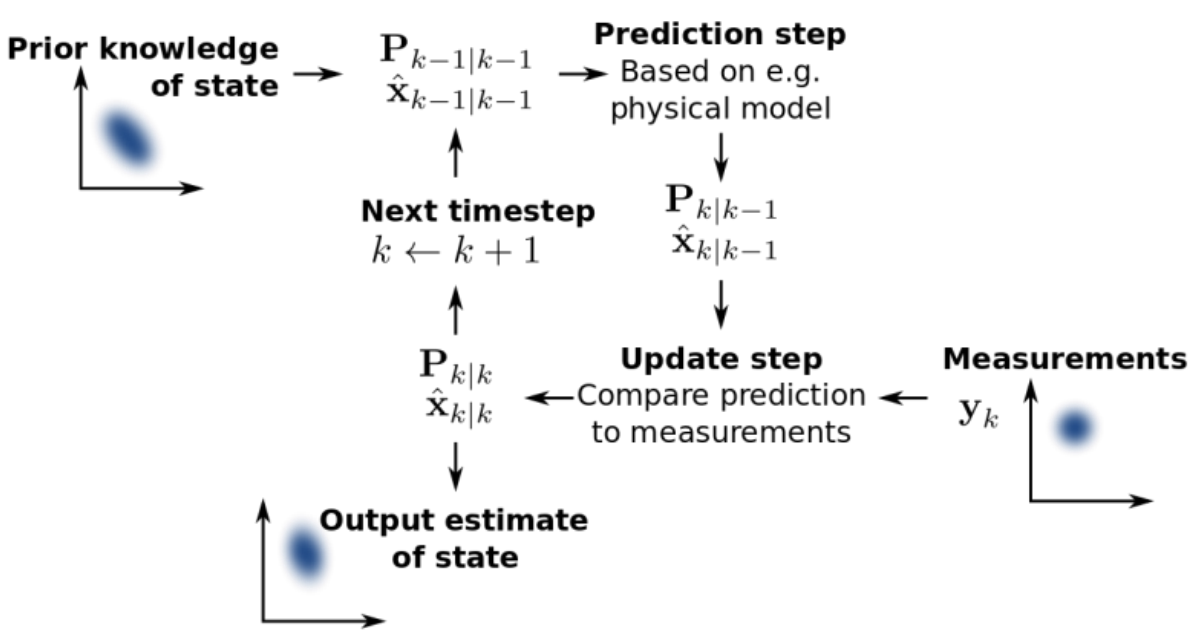

Figure.2 A sample image of how Kalman Filtering gets the coefficients.

Where $\mathrm{i}$ denotes the unspecified number of iterations required to diagonalise the matrix and $\boldsymbol{G}_{i}(z)$ is the elementary paraunitary matrix calculated at iteration i. Each of these matrices consists of a complex elementary scalar rotation matrix, $\boldsymbol{Q}^{(j, k)}(\theta, \phi)$, preceded by an elementary delay matrix, $\boldsymbol{B}^{(k, t)}(z)$ formulated as where at each iteration the parameters $j, k, t, \theta$ and $\phi$ are appropriately chosen depending on [23]

$$
\boldsymbol{G}_{i}(z)=\boldsymbol{Q}^{(j, k)}(\theta, \varnothing) \boldsymbol{B}^{(k, t)}(z)
$$

Where at each iteration the parameters $j, k, t, \theta$ and $\phi$ are appropriately chosen depending on the coefficients within the matrix $\boldsymbol{R}(z)$ that the elementary paraunitary matrix is attempting to annihilate. The matrix will clearly be paraunitary as each term is paraunitary. The two types of elementary paraunitary matrices will now be discussed.

$$
\begin{gathered}
\widehat{\boldsymbol{Q}}^{(j, k)}(\theta, \emptyset)=\left[\begin{array}{cc}
c & s e^{i \emptyset} \\
s e^{-i \emptyset} & c
\end{array}\right] \\
\boldsymbol{B}^{(k, t)}(z)=\left[\begin{array}{ccc}
I_{k-1} & 0 & 0 \\
0 & z^{-t} & 0 \\
0 & 0 & I_{p-k}
\end{array}\right]
\end{gathered}
$$

In the above equations, The elementary scalar rotation matrix $\boldsymbol{Q}^{(j, k)}(\theta, \phi)$, takes the form of a $p \times p$ identity matrix with the exception of the four elements positioned at the intersection of rows $j$ and $k$ with columns $\boldsymbol{j}$ and $k$.

\subsection{Kalman filter}

Linear quadratic estimation (LQE), the other name for Kalman Filtering is an algorithm which using a series of measurements observed over various ranges of time, containing both noise which are statistical as well as measurement and process and other inaccuracies, and produces estimates of unknown variables of the input which is passed through it that tend to be more precise and specific than those based on a single measurement alone. The filter is named after Rudolf E. Kalman, one of the primary developers of its theory.

Fig. 2 explains the kalman filtering and its coefficient generation. The Kalman filter keeps track of the estimated state of the system and the variance or uncertainty of the estimate. The estimate is updated using a state transition model and measurements. $X_{(k \mid k-1)}$ denotes the estimate of the system's state at time step $\mathrm{k}$ before the $\mathrm{k}^{\text {th }}$ measurement has been taken into account; $\mathrm{P}_{(\mathrm{k} k \mathrm{k}-1)}$ is the corresponding uncertainty.

Coming to the calculation using the Kalman filter, the following steps are followed for deriving the coefficients. Initially all the necessary declarations are made like getting the speech, calculating the length, initializing the standard transition matrix, priori or posterior matrix, kalman gain matrix, error etc. all to zero.

$$
K=\operatorname{sig} \times F \times\left(F^{\prime} \times \operatorname{sig} \times F+R\right)^{-1}
$$

Where $\mathrm{K}$ is the Kalman gain, sig is the posterior covariance matrix, $\mathrm{F}$ is the standard transition matrix and $\mathrm{R}$ is the measurement noise.

$$
\begin{aligned}
& \operatorname{sig}=\operatorname{sig}-\operatorname{sig} F \times\left(F^{\prime} \times \operatorname{sig} \times F+R\right)^{-1} \times \\
& \left(F^{\prime} \times \operatorname{sig}\right)+Q
\end{aligned}
$$

Where $\mathrm{Q}$ is the process noise. With these equations, Estimated signal is derived which further is subtracted from the input and the required output is obtained without the acoustic echo. 


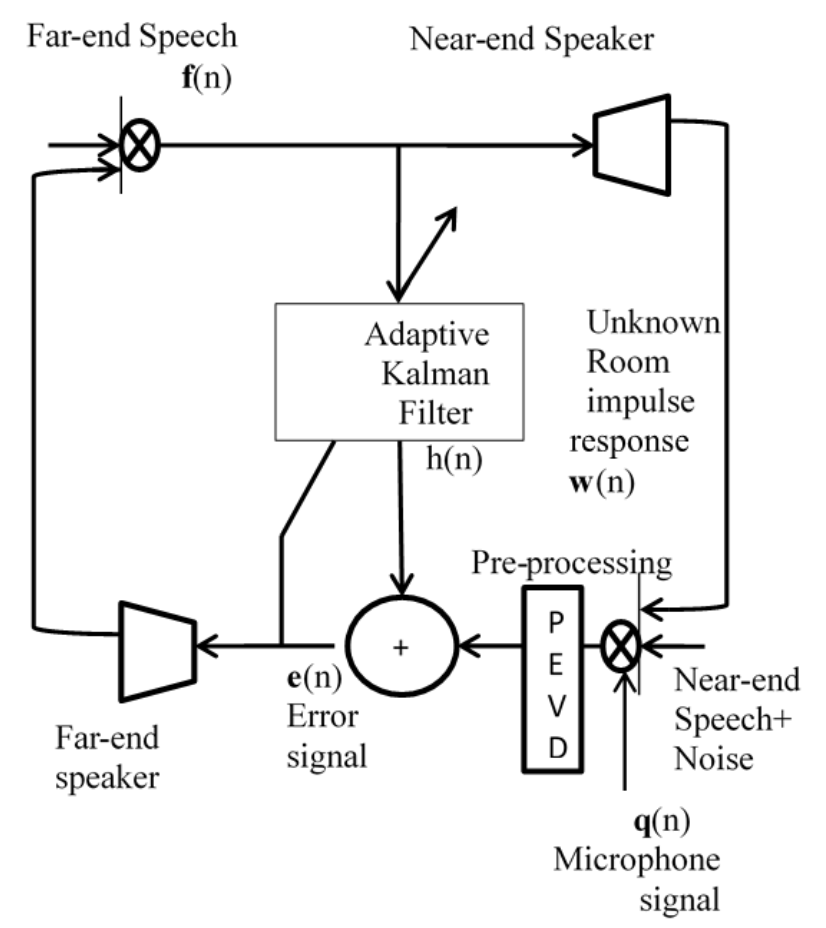

Figure. 3 The block diagram of the proposed system.

\subsection{Proposed PEVD based adaptive kalman filter}

The block diagram of our proposed model is shown in Fig. 3. In Fig. 3 the flow of the working process is shown as per how the echo cancellation will go about. Initially a far end signal is taken and passed through the system. Here itself it is passed through the Kalman Filter to generate/estimate the coefficients with prior knowledge. Now when this signal is played loud in the near end room, the signal gets reflected in the room and gets convolved with the room impulse response. Now when the near end speech will be recorded, this Acoustic Echo along with noise will be added to the near end speech signal. Now this forms the near end speech. This is pre-processed by Polynomial Eigen Value Decomposition which strongly de-correlated the input we give it to it. It also helps in the reduction of the noise. This removed noise signal is tested/played and checked whether the noise is removed or not. Then the Kalman Filter generated/estimated coefficients are subtracted from the Pre-processed signal on an iterative basis. The signal is again passed into the Kalman Filter where the coefficients again get updated and are again subtracted. Then the signal is again tested/ played to check how much echo is removed. Then the Echo Return Loss Enhancement (ERLE) is calculated which is 10 times $\log$ of the output signal to input signal. This measurement is calculated in decibels.
The output is checked and compared with the required output that is $30-40 \mathrm{~dB}$.

\section{Implementation of the proposed method}

\subsection{ERLE (echo return loss enhancement)}

The Echo cancellation performance has been measured in terms of Echo Return Loss Enhancement (ERLE)

$$
E R L E=\operatorname{lolog}_{10} \frac{E\left\{h^{2}(n)\right\}}{E\left\{e^{2} r(n)\right\}}
$$

$E\left\{h^{2}(n)\right\}$ is the power of the original echo and $E\left\{e_{r}^{2}(n)\right\}$ is the power of the residual echo, $e_{r}^{2}(n)=e(n)-s(n)$ is the residual echo, error signal $e(n)$ is the difference between the microphone signal and estimated echo signal, $s(n)$ is the far-end speech signal . From the literature, optimum value of ERLE for a good echo cancellation lies between $30 \mathrm{~dB}-40$ $\mathrm{dB}$. Higher value of ERLE gives better echo cancellation. According to ITU-T recommendation G.167 [2, 5], the value of ERLE should be $30 \mathrm{~dB}$ for hands free telephones during double talk.

\subsection{Simulation procedure}

The simulation was performed in Matlab-2016a and the results were obtained using built in speech (TIMIT database) and real recorded speech. For first experimental setup: TIMIT database is used to obtain far-end signal $\boldsymbol{f}(n)$ and near-end signals $\boldsymbol{v}(n)$ which are sampled at $16 \mathrm{kHz}$ as shown in figure 4 and 5. To create an echo signal $\boldsymbol{h}(n)$ at the microphone, 512-tap impulse response is convolved with far-end signal which simulate a room with reverberation time of $0.8 \mathrm{~s}$. Male voice is used as a far-end speech and female voice is used as near-end speech signal. Both the signals are active during entire duration to create the double talk conditions. The created echo signal mixed with the near-end signal is used as a microphone signal $\boldsymbol{q}(n)$ shown in Fig. 6. Then this was passed through the PEVD preprocessing which strongly de-correlated the input which was given to it and noise was also eliminated. Then kalman coefficients were subtracted from it and the error output was obtained which further was compared with the input of microphone for ERLE. The output was obtained to be approximately 30$40 \mathrm{~dB}$, which is higher efficiency compared to Ayush Jain et al [18]. 


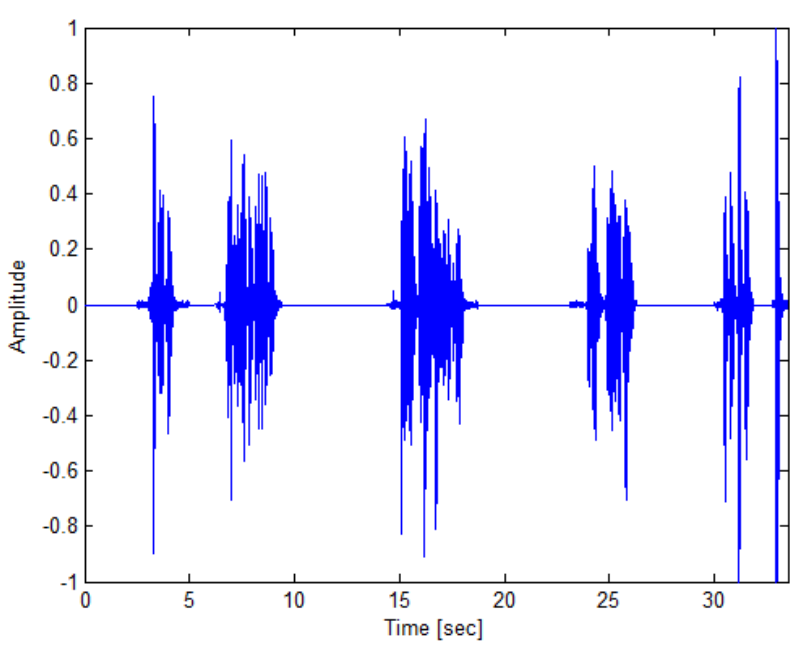

Figure.4 Near-end Speech Signal

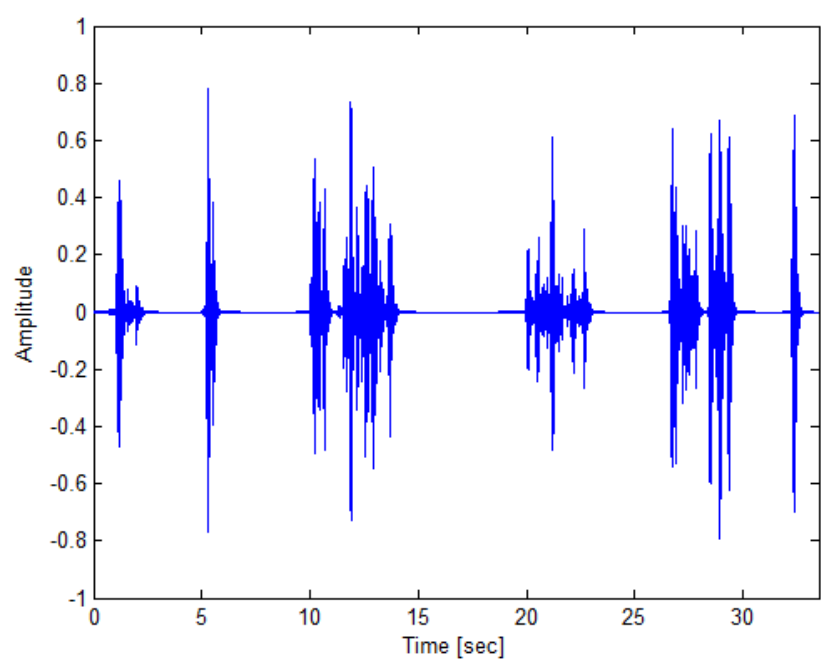

Figure.5 Far-end Echoed Speech Signal

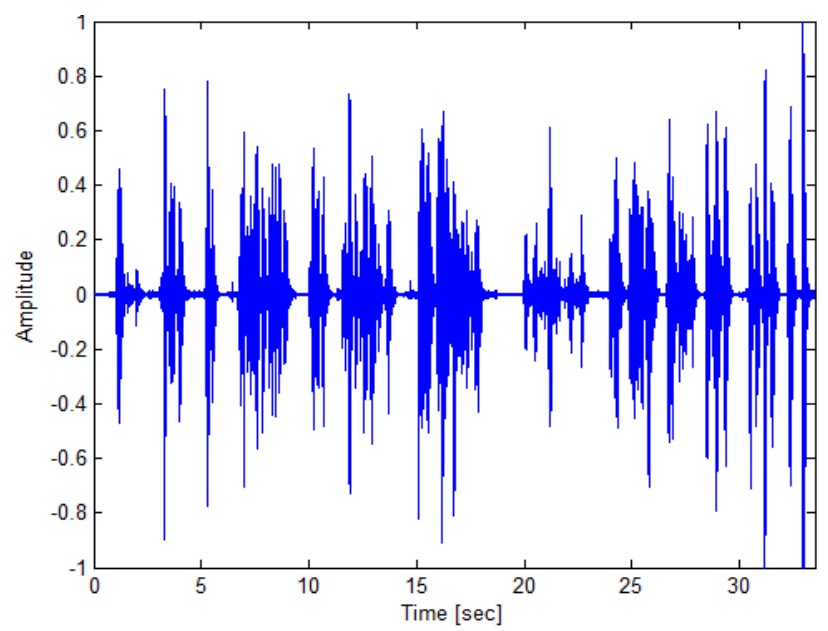

Figure.6 Microphone Speech Signal

\subsection{The simulations without using PEVD pre- processing}

From Fig. 7, the ERLE output it is evident that wherever the echo was present there is a certain amount of output power. This shows that the output we have obtained is proper without echo. According to ITU standards, if the echo return loss enhancement is in the range of $30-40 \mathrm{~dB}$ then the echo cancellation is proper and acceptable. In this setup ERLE lies between 32 to $35 \mathrm{~dB}$. On hearing the output it was clearly evident the far end echo recorded in microphone was cancelled out. In the graph we can also see some $0-15 \mathrm{~dB}$ range of ERLE, these denote the noise in the microphone input or it can even mean the two sounds both near and far have got mixed and being eliminated.

\subsection{The simulation using PEVD pre-processing}

From Fig. 8, power graph is evident yet again that the PEVD pre-processing based acoustic echo canceller also has similar output but a better one. The echo cancelled is actually more reliable and proper. The ERLE value lies between $35-40 \mathrm{~dB}$. In this setup, Kalman filter was set for 3 iterations and then we got the output. In the first iteration, the some echo was cancelled, but by the third iteration

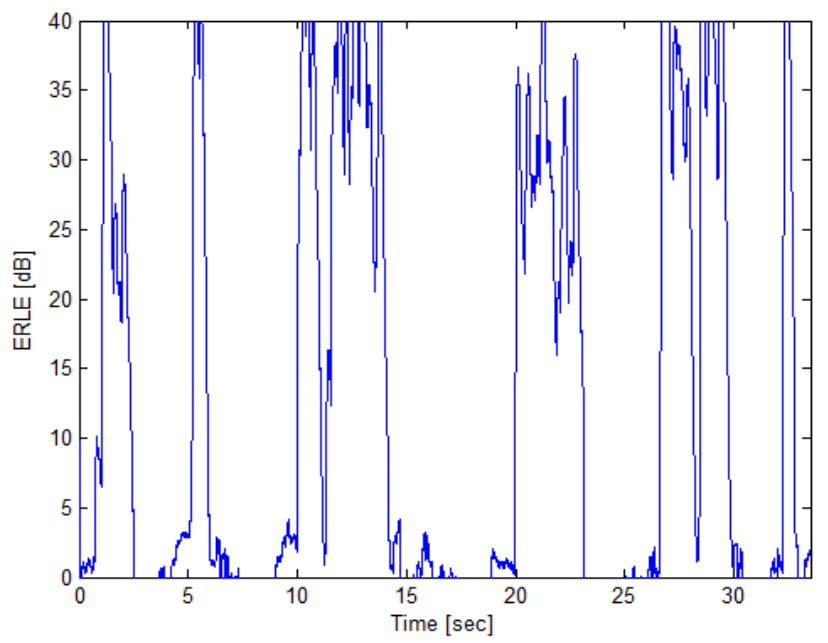

Figure.7 ERLE output

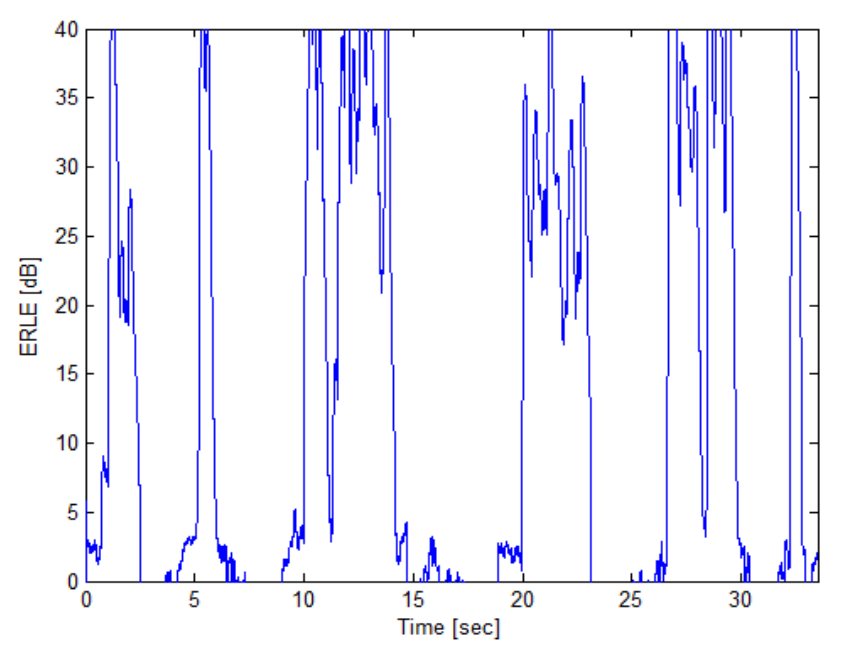

Figure.8 ERLE Output (iteration 3) 
the output obtained was almost perfectly expected one.

\subsection{Simulation using Real time recorded speech}

In this case the voice of the two people conversation is recorded in the super market, which is shown in Fig. 9. Sampling frequency is of 8000 $\mathrm{Hz}$ and duration of the conversation is 3 minutes. From Fig. 9 far-end and near-end recorded speech in the super market is processed by PEVD based kalman filter. The output of the filter results in more than $35 \mathrm{~dB}$ ERLE, which shows that echo is greatly reduced along with noise.

The table 1 gives the final comparison from all the simulation methods discussed in section 4.2-4.5. The result analysis in the table 1 is based on two models, one with only kalman filter and the other with both kalman filter and PEVD pre-processing. Two set of speech signals are used, one is from TIMIT database and other is recorded speech in the noisy environment. The average ERLE of all the section 4.2-4.5 is calculated and shown in the table.
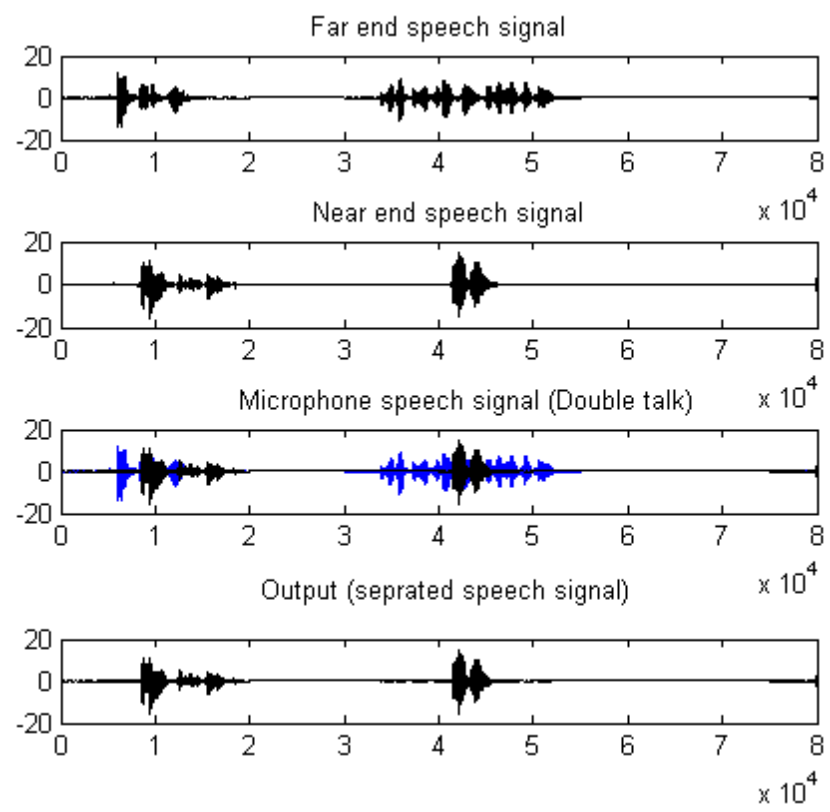

Figure.9 Recorded input speech and output (echo separated speech) in the real time scenario

Table1. Results analysis

\begin{tabular}{|c|c|}
\hline Type of simulation & $\begin{array}{l}\text { Average } \\
\text { ERLE(dB) output }\end{array}$ \\
\hline $\begin{array}{lll}\text { Only } & \text { Kalman } & \text { filter(inbuilt } \\
\text { speech) }\end{array}$ & $34.2 \mathrm{~dB}$ \\
\hline $\begin{array}{l}\text { Kalman filter+ } \\
\text { processing (inbuilt speech) }\end{array}$ & $36.7 \mathrm{~dB}$ \\
\hline $\begin{array}{l}\text { Only Kalman Filter(recorded } \\
\text { speech) }\end{array}$ & $33.6 \mathrm{~dB}$ \\
\hline $\begin{array}{l}\text { Kalman filter+ PEVD pre- } \\
\text { processing (recorded speech) }\end{array}$ & $35.6 \mathrm{~dB}$ \\
\hline
\end{tabular}

From the table 1, PEVD based adaptive kalman filter in both TIMIT database and recorded speech gives higher ERLE of greater than $35 \mathrm{~dB}$ which is good echo cancellation. The range of ERLE outperforms the previous algorithms [18].

\section{Conclusion}

This paper proposes a new PEVD based adaptive Kalman filter to cancel the acoustic echo in a noisy environment. The performance of the echo cancellation is measured using ERLE. From the comparisons plot and higher value of ERLE from the table confirms that the proposed method offers higher cancellation of echo during double talk situation in the presence of noise compared to previous algorithm [18]. With the inclusion of PEVD based transformation to the adaptive kalman filter, the ERLE is further increased to $5 \mathrm{~dB}$, The output of the proposed method is in the range of 35$40 \mathrm{~dB}$ in the echo regions, greater than $35 \mathrm{~dB}$ is good echo cancellation as per ITU standards. On hearing the output generated from the Matlab file, the echo was eliminated almost completely. As the method of kalman filtering has already been designed in the past, the proposed model has a PEVD preprocessing added to it which will help get better accuracies in case of the echo cancellation as well as the noise cancellation or the noise removal. Implementing the above method in real time processor will help to reduce the execution time.

\section{Acknowledgments}

I thank my guide and my students who helped in all aspects for successful completion of this paper. I thank my family for support me to submit this paper.

\section{References}

[1] B. Widrow, and M. E. Hoff, "Adaptive switching circuits", IRE Wescon Conv. Rec., pp.96-104, 1960.

[2] D. L. Duttweiler, "A twelve-channel digital echo canceller", IEEE Transaction on Communication, Vol.26, pp.647-653, 1978.

[3] M. M. Sondhi, "An adaptive echo canceller", Bell syst. Tech Journal, Vol.46, No.3, pp.497510, 1967.

[4] M. M. Sondhi, D. R. Morgan, and J. L. Hall, "Stereophonic acoustic echo cancellation-an overview of the fundamental problem", IEEE Signal Processing Letters, Vol.2, No.8, pp.148151, 1995.

[5] H. Ye, and B. X. Wu, "A new Double talk detection algorithm based on the orthogonality 
theorem", IEEE Trans. Commun. Vol.39, No.11, pp.1542-1545, 1991.

[6] T. Gansler, "A double-talk detector based on Coherence", IEEE Trans. Commun., Vol.44, No.11, pp.1421-1427, 1996.

[7] H. Buchner, J. Benesty, T. Gansler, and W. Kellermann, "Robust extended multi delay filter and double-talk detector for acoustic echo cancellation", IEEE Trans. Audio, Speech, Lang. Process. Vol.14, No.5, pp.1633-1644, 2006.

[8] J. H. Cho, D. R. Morgan, and J. Benesty "An objective technique for evaluating double talk detectors in acoustic echo canceller", IEEE Trans. Speech Audio processing. Vol.7, No.11, pp.718-724, 1999.

[9] J. Benesty, D. R. Morgan, and J. Cho, "A new class of doubletalk detectors based on crosscorrelation", IEEE Trans. Speech Audio Process. Vol.8, No.3, pp.168-172, 2000.

[10] T. Gansler, and J. Benesty, "A frequencydomain double-talk detector based on a normalized cross-correlation vector", Signal Processing, Vol.81, pp.1783-1787, 2001.

[11] J. C. Jenq, and S. F. Hsieh, "A double talk resistant echo cancellation based on the iterative maximal-length correlation". In Proc. Int. Symp. Circuits Syst., pp.237-240, 2000.

[12] J. Y. Tourneret, N. B. Bershad, and C. Bermudez, "Echo Cancellation-The Generalized Likelihood Ratio Test for DoubleTalk Versus channel change", IEEE Trans., on Signal Processing, Vol.57, No.3, pp.916-926, 2009.

[13] K. H. Lee, J. H. Chang, N. Kim, S. Kang, and Y. Kim, "Frequency domain double-talk detection based on the Gaussian mixture model”, IEEE Signal Processing, Vol.17, No.5, pp.453-456, 2010.

[14] R. E. Kalman, "A new approach to linear filtering and prediction problems", J. Basic Engg., Vol 82, pp.35-45, March, 1960.

[15] G. Enzner, and P. Vary, "Frequency-domain adaptive kalman filter for acoustic echo control in hands-free telephones", Signal Processing, Vol.86, No.6, pp.1140-1156, 2006.

[16] S. Tanan, K. Nathwani, A. Jain, R. Rani, A. Tripathy and R. M. Hegde, "Acoustic Echo and noise cancellation using kalman filter in a modified gsc frame work", In: Proc. of the 2014 conference record of the forty six Asilomar conference IEEE on signals systems and computers.

[17] P. Constantin, J. Benesty, and C. Silviu: "widely linear general kalman filter for stereophonic acoustic echo cancellation", Signal processing, Vol. 94, pp.570-575, 2014.

[18] A. Jain, G. Sanchit, K. Nathwani, M. Rajesh, and R. M. Hegde, "Robust acoustic echo cancellation using kalman filter in double talk scenario", Speech Communication, Vol. 70, pp.65-75, 2015.

[19] R. Soydan, J. G. McWhirter, and W. Stephan, "Design of FIR Para unitary Filter Banks for Sub band Coding Using a Polynomial Eigenvalue Decomposition", IEEE Trans. on signal processing., Vol.59, No.11, pp.52535264, 2011.

[20] K. Mohanaprasad, and P. Arulmozhivarman, "Wavelet based adaptive filtering algorithms for acoustic noise cancellation". International Review on Computers and Software, Vol. 9, No.10, pp.1675-1681, 2014.

[21] K. Mohanaprasad, and P. Arulmozhivarman, "Wavelet based ICA using Maximization of Non-Gaussianity for Acoustic Echo Cancellation during Double talk situation", Applied Acoustics, Vol.97, pp.37-45, 2015.

[22] K. Mohanaprasad and P. Arulmozhivarman, "Wavelet-Based ICA Using Maximum Likelihood Estimation and InformationTheoretic Measure for Acoustic Echo Cancellation during Double Talk Situation", Circuits Systems and Signal processing, Vol.34, No.12, pp.3915-3931, 2015.

[23] T. Chi Hieu, and W. Stephan, "shortening the order of paraunitary matrices in SBR2 algorithm", In: Proc. Of 2007 6th international conference on Information, Communications and Signal Processing, Singapore, pp.10-13, December, 2007.

[24] J. Foster, J. G. Mcwhirter, and J. Chambers, "Limiting the order of polynomial matrices within the SBR2 algorithm", In: Proc. of $7^{\text {th }}$ International Conference on Mathematics of Signal Processing, Cirencester, 2006. 